\title{
Hot Stars with Winds: The CMFGEN Code
}

\author{
D. John Hillier \\ University of Pittsburgh, Department of Physics and Astronomy, \\ 100 Allen Hall, Pittsburgh, PA 15260, USA \\ email: hillier@pitt.edu
}

\begin{abstract}
CMFGEN is an atmosphere code developed to model the spectra of a variety of objects - O stars, Wolf-Rayet stars, luminous blue variables, A and B stars, central stars of planetary nebula, and supernovae. The principal computational aim of CMFGEN is to determine the temperature and ionization structure of the atmosphere, and the atomic level populations. Toward this end, we have developed several different radiative transfer modules that (a) solve the transfer equation for spherical geometry in the comoving frame, (b) solve the static transfer equation in the plane parallel approximation without, or with, a vertical velocity field, (c) solve the static transfer code for a spherical atmosphere allowing for all relativistic terms, (d) solve the time-dependent spherical transfer equation to first order in $v / c$ for a homologous expansion, and (e) solve the time-dependent spherical transfer equation allowing for all relativistic terms. To achieve consistency between the radiation field, temperature structure, and level populations we use a linearization technique. Line blanketing is accurately treated while complex photoionization cross-sections, containing numerous resonances, can also be handled. Spectra, for comparison with observation, are computed using CMF_FLUX. Several other auxiliary programs have also been developed - these include diagnostic tools as well as programs that allow the effect on spectra of rotation and departures from spherical geometry to be investigated. In this presentation we will briefly describe CMFGEN and auxiliary codes.
\end{abstract}

Keywords. stars: atmospheres, emission line, line: formation

\section{Introduction}

CMFGEN is a model atmosphere code originally developed to study the spectra of massive stars, although it can also be used to study a variety of other objects. A common feature of massive stars is that they exhibit stellar winds. These stellar winds are driven by radiation pressure (acting through bound-bound transitions in the UV and EUV). Because of the stellar winds, the atmospheres are not plane-parallel - the simplest geometry we can adopt is spherical geometry. Further, because of the velocity field associated with the wind, it is more convenient to solve for the radiation field in a frame moving with the gas - the comoving frame. In this frame, the opacities and emissivities can be assumed to be isotropic. Also, because of the intense radiation fields, and low photosphere and wind densities, we cannot assume Local Thermodynamic Equilibrium (LTE) to evaluate atomic and ion level populations. Rather, the atmospheres are said to be in non-LTE, and we have to solve the statistical equilibrium equations (which describe rates into, and out of, each atomic/ion level). Because of the strong coupling between the radiation field and the level populations, simple iterative techniques cannot be used.

\section{CMFGEN}

The basic purpose of CMFGEN is to determine the star's atmospheric structure: the temperature and ionization structure, and the atomic level populations for all atoms/ions. 
CMFGEN simultaneously solves the radiative transfer equation (RTE) for spherical geometry in the comoving frame in conjunction with the statistical equilibrium equations (SEEs) and radiative equilibrium equation (REE) (Hillier 1990; Hillier \& Miller 1998). Since the equations are non-linear and coupled - the radiation field depends on the level populations which in turn depend on the radiation field - an iterative technique must be used. In CMFGEN we linearize the RTE which allows us to solve for the $\delta J_{\nu}$ (the linearized mean intensity) as a function of the (unknown) corrections to the opacities and emissivities, and hence as a function of the unknown correction $(\delta n)$ to the populations (and temperature and electron density). We then eliminate the $\delta J_{\nu}$ from the linearized SEEs and REE to obtain a set of simultaneous equations in the $\delta n$. In eliminating the $\delta J_{\nu}$, we only retain the influence of the local populations (diagonal operator), or that of populations at the local and neighboring depths (tridiagonal operator). With the diagonal operator, we have a set of $N_{\mathrm{V}}$ simultaneous equations at each depth, where $N_{\mathrm{V}}$ is the total number of unknown populations (for all species) at each depth. For the tridiagonal operator, we have a block-tridiagonal system (with each block $N_{\mathrm{V}} \times N_{\mathrm{V}}$ ) which is solved efficiently using the Thomas algorithm. For reasons of stability, we rescale the simultaneous equations so that we solve for $\delta n / n$ (Hillier 2003). The technique yields similar convergence properties to approximate lambda operators (see Hillier 1990).

At present, the mass-loss rate and the velocity law above the sonic point must be specified when modeling stellar winds. The structure below the sonic point is obtained by solving the equation of hydrostatic equilibrium.

As CMFGEN has evolved we have developed several different radiative transfer modules:

(a) A module to solve the transfer equation for spherical geometry in the comoving frame: this is the mode used when studying massive stars and their stellar winds.

(b) A module to solve the static transfer equation in the plane parallel approximation.

(c) A module to solve the transfer equation in the plane parallel approximation with a vertical velocity field.

(d) A module to solve the static transfer code for a spherical atmosphere allowing for all relativistic terms.

(e) A module to solve the time-dependent spherical transfer equation to first order in $v / c$ for a homologous expansion.

(f) A module to solve the time-dependent spherical transfer equation for all orders in $v / c$ (undertaken by a graduate student, Chendong Li).

The latter three modules were developed to facilitate the analysis of supernova (SN) spectra.

\section{Applications of CMFGEN}

CMFGEN has been used to determine fundamental stellar parameters, and investigate the stellar winds of Wolf-Rayet (W-R) stars (e.g., Herald et al. 2001), Luminous Blue Variables (LBVs) (e.g., Groh et al. 2009), CSPNs (e.g., Marcolino et al. 2007), O Stars (e.g., Martins et al. 2002; Hillier et al. 2003; Bouret et al. 2003) and A and B stars. It is also being used to study Type I and Type II SN ejecta (e.g., Dessart \& Hillier 2010; Dessart et al. 2011; Dessart \& Hillier 2011). CMFGEN has many uses:

(a) To provide accurate abundances and stellar parameters for comparison with evolution calculations.

(b) To provide EUV radiation fields for input to nebula photoionization calculations.

(c) To provide "limb darkening laws" for interferometry (see, e.g., Figure 1). 


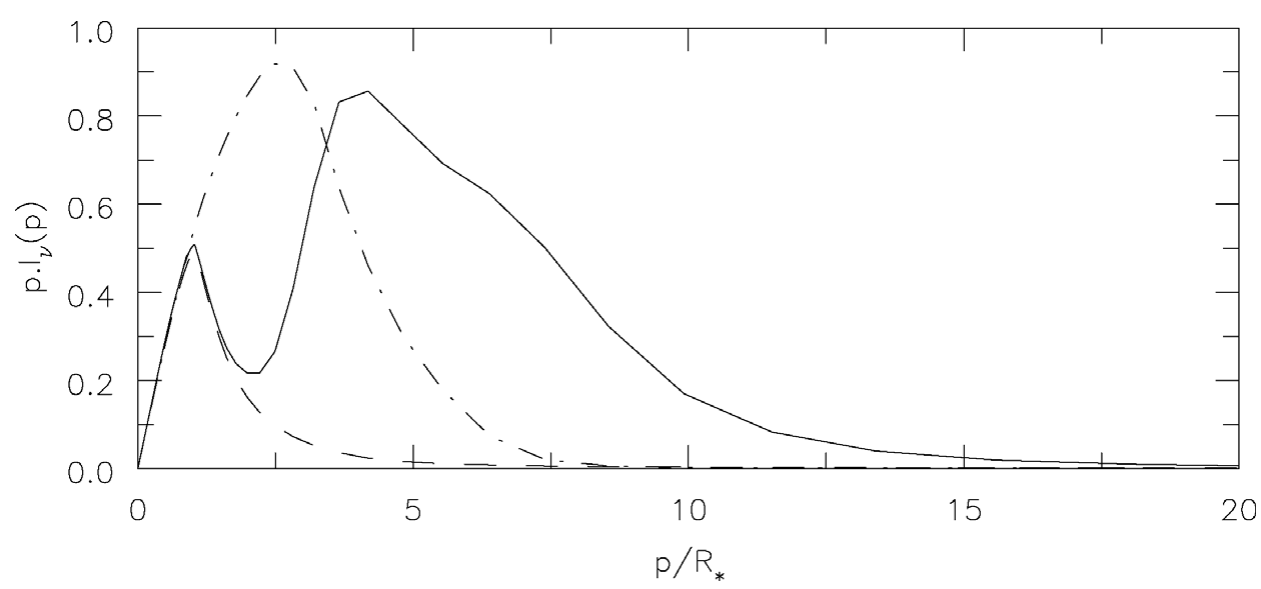

Figure 1. Illustration of $p I_{\nu}(p)$ at 3 wavelengths for a $\gamma^{2}$ Velorum (WC8)-like model with an effective temperature of $\sim 52,500 \mathrm{~K}$. Shown is $p I_{\nu}(p)$ at line center for C III $\lambda 5696$ (solid line), the continuum ( $\lambda 5750$; dashed) and near line center for the C IV $\lambda \lambda 5801,5812$ (dash-dot) doublet. Notice how the line formation regions are more extended than the line emitting region. The dip in $p I_{\nu}(p)$ for C III $\lambda 5696$ is a consequence of the ionization structure.

(d) To provide data for the study of starbursts, star formation in galaxies, etc.

(e) To provide a better understanding of the hydrodynamics of winds.

(f) To assist in the development and testing of approximate methods that can be used in more complex geometries and inhomogeneous media.

(g) To derive distances to Type II SNe using the expanding-photosphere method (EPM), and variants thereof.

(h) To allow SN spectra to be used as an evolutionary and explosion diagnostic tool.

\section{Line Formation in Winds}

While CMFGEN can be run without understanding all of the complex physics involved, a rudimentary knowledge of the basic mechanisms of continuum and line formation in stellar winds is useful if one is to interpret model results, and to understand the sensitivities of various lines to different model parameters. Many different processes contribute to the formation of emission lines in stellar winds, and some of these, with examples, are summarized in Table 1 . In some cases more than one mechanism may contribute, while in other cases (as you go closer to LTE, for example) it may not be possible to determine a specific mechanism. Further information on these processes, with references, can be found in Hillier (2011).

\section{CMF_FLUX}

For the computation of the observed spectrum, we use the comoving frame/observer's frame code CMF_FLuX (Busche \& Hillier 2005). This code was designed to allow the accurate computation of observed spectra with full allowance for different types of broadening mechanisms (e.g., Doppler, Stark, Voigt). To facilitate spectral interpretation, we can compute spectra where we include only selected species, or alternatively, omit selected species (e.g., Fe II). This is particularly useful for examining the direct influence of lines from a given species on the observed spectrum.

The code has two parts. First, as in CMFGEN, we solve the radiative transfer equation in the comoving frame. To treat incoherent electron scattering, arising from the thermal 
Table 1. Summary of mechanisms producing emission lines.

\begin{tabular}{ll}
\hline Mechanism & Example \\
\hline Recombination & H, He emission lines; most C lines in WC stars \\
Dielectronic recombination & N III $\lambda 4640$ triplet in Of stars (Mihalas et al. 1972); \\
& C III $\lambda 2296, \lambda 6740 ;$ many C I lines in WC stars \\
Resonance scattering & P Cygni profiles in UV spectra of O stars; Na I D lines in \\
& LBVs \\
Collisional excitation & C IV $\lambda \lambda 1548,1551$ doublet in WC stars; N IV $\lambda 1486$ in \\
Continuum fluorescence & WN stars; C III $\lambda 1909$ in WC stars \\
& metal lines (Mg II, Si II, Fe II) in P Cygni type stars \\
Raman scattering & $\lambda \lambda 6830,7088$ lines in symbiotic stars (Schmid 1989) \\
Bowen fluorescence & \\
\hline
\end{tabular}

motions of the electrons, we perform several iterations - two are sufficient for O stars. This calculation provides emissivities, opacities, and mean intensities for the observer's frame calculation.

Second, an observer's frame calculation is performed using the usual $(p, z)$ co-ordinate system. Along each ray, we choose a fine spatial grid such that $\Delta V<0.25 V_{\text {Dop }}$ to allow adequate sampling of the rapidly varying opacities and emissivities. On this revised spatial grid, we map the comoving-frame opacities and emissivities - this requires interpolation in both space and in frequency. Along each ray, we perform a formal integration to compute $I_{\nu}(p)$, and from the $I_{\nu}(p)$ we can compute the observed flux using standard numerical quadrature. Optionally, the $I_{\nu}(p)$ (effectively giving the limb darkening law), can also be output.

\section{Diagnostic Tools/Files}

CMFGEN is a complicated code, with many different capabilities and control options. While care has been taken developing the code, it uses many different numerical techniques and approximations. These techniques and approximations may only work in a limited parameter range, and it is important that users perform checks to ensure code accuracy when moving to different parameter ranges. CMFGEN, like all complicated codes, should not be used as a black box.

To facilitate checking and interpretation of results, we have developed two display packages, DISPGEN and PLT_SPEC. The first, DISPGEN, is designed to investigate the structure of the model atmosphere. It allows populations and non-LTE departure coefficients, the ionization structure, temperature structure, line and continuum opacities, and optical depth scales, for example, to be examined. PLT_SPEC is used for comparing model spectra with observation.

In addition to the display packages, many diagnostic files are created. For example, for every ionization stage we create a file which lists photoionization rates and recombination rates for each level, and the recombination coefficient, as a function of depth. Another file contains a check for the electron energy balance. As noted by Hillier (2003), the assumptions of radiative equilibrium and electron energy balance are related by the SEEs. In the presence of super levels they are not equivalent, and an examination of the errors in the electron energy balance provides a check on both program coding, and inaccuracies introduced by the use of super levels.

Another file, OBSFLuX, provides the observed spectrum, and the luminosity as a function of depth. The latter also provides a check on code consistency. In stellar models, the 
luminosity is specified only at the lower boundary - its constancy with depth follows only when radiative equilibrium is satisfied at each depth.

STEQ_VALS lists the current error in the SEEs and the fractional corrections $(\delta n / n)$ for each population at each depth. The latter is particularly useful for investigating convergence issues.

\section{Obtaining the code}

CMFGEN is available at http://kookaburra.phyast.pitt.edu/hillier. Register to receive emails with updated information about CMFGEN. Also available, at the same website, is documentation, and a small grid of models that provides test cases and starting models for use with CMFGEN.

\subsection{Technical Support}

CMFGEN is a complex code, and it takes time to understand all its features and nuances. New users should first read the supplied manual to at least familiarize themselves with some of the basic philosophies behind the code. When problems occur, check the input files for errors, check OUTGEN for diagnostic messages, and the manual for possible solutions. If you are unable to solve the problem, you can email me for assistance. If needed, you can also visit me in Pittsburgh. Base models for $\mathrm{O}$ stars and for some $\mathrm{W}$-R stars are on my website. There are numerous CMFGEN models around, so ask me, or other CMFGEN users for access to these models.

I appreciate feedback. If you find an error, it is absolutely essential that you inform me so that other users are not also affected. Suggestions for improvements are also welcome.

\section{Acknowledgements}

DJH gratefully acknowledges support from STScI theory grant HST-AR-11756.01.A and NASA theory grant NNX10AC80G.

\section{References}

Bouret, J.-C., Lanz, T., Hillier, D. J., Heap, S. R., Hubeny, I., Lennon, D. J., Smith, L. J., \& Evans, C. J. 2003, ApJ, 595, 1182

Busche, J. R. \& Hillier, D. J. 2005, AJ, 129, 454

Dessart, L. \& Hillier, D. J. 2010, MNRAS, 405, 2141

-. 2011, MNRAS, 410, 1739

Dessart, L., Hillier, D. J., Livne, E., Yoon, S.-C., Woosley, S., Waldman, R., \& Langer, N. 2011, MNRAS, 414, 2985

Donati, J.-F., Babel, J., Harries, T. J., Howarth, I. D., Petit, P., \& Semel, M. 2002, MNRAS, 333,55

Georgiev, L. N., Hillier, D. J., \& Zsargó, J. 2006, A\& A, 458, 597

Groh, J. H., Hillier, D. J., Damineli, A., Whitelock, P. A., Marang, F., \& Rossi, C. 2009, ApJ, 698,1698

Herald, J. E., Hillier, D. J., \& Schulte-Ladbeck, R. E. 2001, ApJ, 548, 932

Hillier, D. J. 1990, A\&A, 231, 116

Hillier, D. J. 2003, in Astronomical Society of the Pacific Conference Series, Vol. 288, Stellar Atmosphere Modeling, ed. I. Hubeny, D. Mihalas, \& K. Werner, p. 199

-. 2011, ApESSS, (in press)

Hillier, D. J., Lanz, T., Heap, S. R., Hubeny, I., Smith, L. J., Evans, C. J., Lennon, D. J., \& Bouret, J.-C. 2003, ApJ, 588, 1039

Hillier, D. J. \& Miller, D. L. 1998, ApJ, 496, 407

Kurosawa, R., Hillier, D. J., \& Pittard, J. M. 2002, A\&A, 388, 957 
Luehrs, S. 1997, PASP, 109, 504

Marcolino, W. L. F., Hillier, D. J., de Araujo, F. X., \& Pereira, C. B. 2007, ApJ, 654, 1068

Martins, F., Schaerer, D., \& Hillier, D. J. 2002, A\&A, 382, 999

Mihalas, D., Hummer, D. G., \& Conti, P. S. 1972, ApJL, 175, L99+

Schmid, H. M. 1989, A\& A, 211, L31

Wade, G. A., Fullerton, A. W., Donati, J.-F., Landstreet, J. D., Petit, P., \& Strasser, S. 2006, $A \mathscr{S} A, 451,195$

\section{Discussion}

O. De Marco: Stellar winds are disturbed by companion stars. Can wind models model the effects of this disturbance on the line profiles? Have such efforts already been undertaken?

J. Hillier: An obvious manifestation of binarity in $\mathrm{O}$ stars is wind-wind collisions which produce hard X-rays. Phase-locked profile variability is also seen, especially in $\mathrm{O}$ star and W-R systems (e.g., V444 Cygni). A semi-quantitative description of the influence of the interaction region on line profiles is provided by Luehrs (1997). Limited modeling of wind-wind collision processes is being attempted by various groups but the multidimensional nature (at least 2D) makes it a difficult task. For example, Kurosawa et al. (2002) used a Monte-Carlo code, in conjunction with 1D CMFGEN models, to interpret the line-profile and polarization variations in V444 Cygni. With the advent of improved hydrodynamic models, which can predict the structure of the interaction region, the demand for improved modeling of interacting binary systems will increase.

M. Montgomery: Have you considered modeling oblate stars for rapid rotators as you consider spherical geometry?

J. Hillier: To fully model rapid rotators with stellar winds, we would need to go to $2 \mathrm{D}$. Going to $2 \mathrm{D}$ is computationally expensive, requiring $>10^{3}$ times the computational effort for a model of similar complexity to a 1D CMFGEN model (due to the extra spatial dimension and the loss of symmetry in the radiation field). For stars without winds, the plane-parallel approximation is valid, and we can create spectra by tiling the surface of the rapidly rotating star with the appropriate model atmospheres. I have a code to do this - it was developed by an undergraduate student, Charles Warren. For stars with winds, it is a little more difficult because we now have the additional variation of the (uncertain) wind properties with latitude. For slow/moderate rotators, we have a 2D synthesis code (Busche \& Hillier 2005) where we can apply latitude scaling laws (or potentially string a series of $1 \mathrm{D}$ models together) to investigate the influence of rotation. Finally, I note that we have developed a 2D code (Georgiev et al. 2006), but it is much less sophisticated than CMFGEN.

J. Freimanis: What about the inclusion of magnetic fields, Zeeman effect, Hanle effect, and so on, into your models?

J. Hillier: Most $O$ stars have only very weak magnetic fields $(<100 G)$, and these are probably not crucial for understanding their spectra. However a few $\mathrm{O}$ stars have very intense magnetic fields [e.g., $\theta^{1}$ Orionis C, $B>1 \mathrm{kG}$; Donati et al. (2002)]. In these cases the magnetic field will control the flow of wind material from the star's surface producing shocks, etc. In such cases $2 \mathrm{D}$ or $3 \mathrm{D}$ modeling is required, which, as noted above, is difficult. However, researchers have successfully shown that an oblique rotator model can match the emission-line variability seen in $\theta^{1}$ Orionis $\mathrm{C}$ (Wade et al. 2006). 